\title{
Research on the Application of Goal Teaching Method in Applied Undergraduate Teaching
}

\author{
Huang wen ${ }^{1, \mathrm{a}}$

\begin{abstract}
${ }^{I}$ Southwest Jiaotong University Hope College Electromechanical and Rail Vehicle Engineering Department Jintang Chengdu
\end{abstract} \\ a522818158@qq.com
}

\begin{abstract}
Application-oriented colleges and universities take "application-oriented talents" as their training goal, Guided by solving practical problems on site,Cultivate students' practical ability and practical ability to solve problems.In this paper, the application of target teaching method in application-oriented undergraduate colleges is studied, and various teaching methods are used flexibly and reasonably and efficiently.Stimulate students' learning enthusiasm, To cultivate high quality,Application-oriented talents who meet the needs of society make contributions.
\end{abstract}

Keywords: application-oriented undergraduate course, application-oriented Talents, target teaching method, comprehensive quality

\section{目标教学法在应用型本科教学中的应用研究}

黄 文 $^{1, a}$

${ }^{1}$ 西南交通大学希望学院 机电与轨道车辆工程系 金堂 成都
${ }^{2} 522818158$ @qq.com

摘要

应用型本科院校是以 “应用型人才” 为培养目标, 以能解决现场实际问题为导向, 培养学生的动手能力, 解决 问题的实践能力。本文对目标教学法在应用型本科院校中的应用进行研究，合理、高效的将各种教学手段灵活 运用起来, 激发学生的学习热情, 为应用型本科院校培养出高质量、适应社会需求的应用型人才做出贡献。

关键词：应用型本科；应用型人才；目标教学法；综合素质

\section{1. 应用型本科培养目标}

高等教育法明确规定: 高等教育必须贯彻国家的 教育方针, 为社会主义建设服务, 与生产劳动相结合, 使受教育者成为德、智、体等方面全面发展的社会主 义事业的建设者和接班人。高等教育的任务是培养具 有创新精神和实践能力的高级专门人才, 发展科学技 术文化, 促进社会主义现代化建设 ${ }^{[1]}$ 。应用型本科是 介于研究型和高职高专型的一种高等教育类别。应用 型本科是对传统教学模式下的课程体系、教学模式、 实践机制、教学管理等方面进行改革, 更新教学内容、 教学环节、教学方法和教学手段, 注重实践能力、应 用能力、创新创业能力的培养, 以培养 “应用型人才”
为教育目标, 满足和适应区域经济发展需要, 培养具 有较强社会适应能力和竞争能力的高素质应用型人 才。

\section{2. 传统教学模式的弊端}

传统教学法以传授知识为教学目标, 主要以教师 为中心, 通过讲授的形式将知识传授给学生, 目标单 一, 缺乏对学生能力的培养, 人格目标、情感目标缺 失。传统教学法可以让学生学到较为系统、扎实的专 业基础知识, 但学生长期在这种灌输式的教学模式下 学习, 缺失应有的学习过程一存疑、选择、批判、探索、 想象、创造等，容易形成思维惰性，习惯被动接受，不 积极主动思考问题，更不具备将所学理论知识转化为 
解决实际问题的能力。传统教学法没有对学生学习的 主动性和创造性思维有意思地进行培养, 更没有对学 生人格、情感等素质进行锻炼。长此以往造就了高分 低能的学生。

\section{3. 目标教学法的特色}

目标教学法以理论为实践服务为指导思想, 注重 知识的实用性, 倡导教学过程中师生间的互动。目标 教学法是以教学目标为核心和主线实施课堂教学的 方法, 以教师为主导、以学生为主体的教学方法。教 师以教学目标为导向, 在整个教学过程中围绕教学目 标运用各种教学方法和教学手段展开一系列教学活 动 ${ }^{[2]}$ 。有目标的学习效率是盲目学习效率的数倍, 在 进行教学之前, 向学生展示教学目标, 让学生带着目 标和问题进行学习, 可以激发学生学习的兴趣。同时 目标教学法注重知识趣味性和实用性; 目标教学法注 重学生能力的培养, 教学过程中教师提出问题, 引导 思考, 展开讨论, 充分发挥学生的主体作用, 使学生 在学习讨论中获取知识, 培养自学能力和独立思考的 能力。在课程设置中增加实践、实验性教学环节, 培 养学生的动手能力, 让学生在动手操作过程中, 增长 知识和才干; 目标教学法注重学生人格、情商的培养, 在教学过程中通过师生的交流、同学的讨论、实践过 程中的合作, 可以培养学生的相互尊敬、相互包容、 团结协作、积极向上的精神。

目标教学法按照教学大纲设定教学目标, 通过精 讲点拨, 启发诱导, 问答指导, 讨论交流, 实践操作、 自主学习等教学活动, 来克服学生思维惰性, 变 “要 我学” 为 “我要学”。给学生一个自由思考的空间, 提 供充分发挥想象力和创新能力的学习平台 ${ }^{[3]}$, 让他们 充分感受到学习成功的喜悦和成就感, 激发学生学习 兴趣, 促使学生更加努力地学习。

“目标教学法” 的教学模式: 教学目标的设定 $\rightarrow$ 教学目标的实施 $\rightarrow$ 教学目标的检验与评价 $\rightarrow$ 教学反 馈。这一模式具有较强的可操作性, 该模式的重点在 教学目标的设定, 关键在教学目标的实施。通过教学 评价和教学反馈, 完善教学目标, 改善教学方法, 提 高教学水平。

\section{4. 教学目标的设定}

教学目标分为三个层次: 知识目标, 能力目标和 情感目标。知识目标是基础, 是对所学理论知识的掌 握。能力目标是提升, 是对所学知识的整合、运用能 力, 以及自学能力和动手能力等的提升。情感目标是 对学生综合素质的要求, 是在思想意识, 毅力, 团结 协作, 组织力, 领导力等方面的要求。三个目标不是 孤立的, 是相互融合、相互贯穿为一个整体。教学中 结合教学内容有意识、有目的对三个目标进行设计。

教学目标的设定都是通过上该课程的所有教师 集体讨论研究决定, 通过教学实践不断修改完善。目
标制定上要充分体现应用型本科的特点，做到理论够 用, 加强实践教学，注重能力的培养。下图为目标设 定树状图。

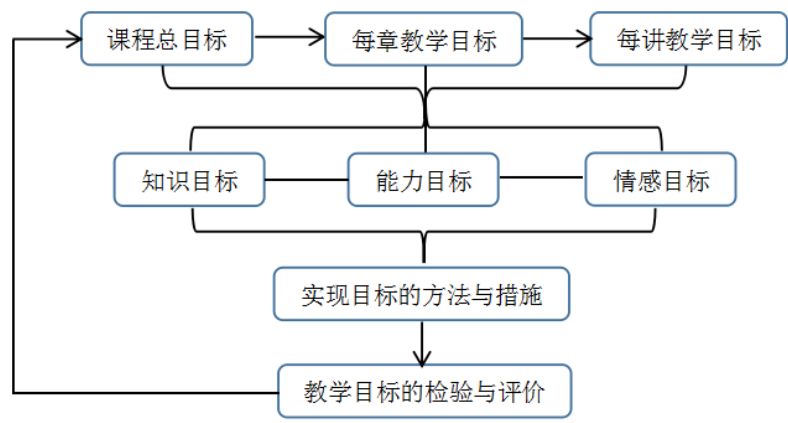

图 1 目标设定树状图

目标教学法要求教师对教材的内容了如指掌，对 课程的知识体系非常清晰, 明确重点、难点。能合理、 科学地设置教学目标, 充分体现三个层次。合理分配 教学时间, 有实现目标的具体方法和措施。在教学实 施过程中, 对各种教学方法和教学手段运用娴熟, 有 非常强的导向和把控能力, 能根据学生的状况及时调 整教学方法, 达到最佳的教学效果。

\section{5. 教学目标的实施}

教学过程将围绕教学目标展开, 是目标教学法中 最关键的一个环节。根据教学内容的特点采用相应的 教学方法和手段来实现教学目标, 不拘泥于一种教学 方法和教学手段, 以学生容易接受, 愿意参与, 积极 主动思考为原则。教学目标的实施过程是通过教学目 标的导入, 教学目标的展开, 教学过程的把控来进行 的。

\section{1. 教学目标的导入}

俗话说: 良好的开头是成功的一半, 上课也是这 样, 好的导入就像磁石一样会把学生牢牢地吸引住, 唤起学生的学习热情, 激发学生的潜在求知欲 [4]。

教学目标的导入可以通过复习旧知识导入, 利用 故事创设情境导入, 图片导入, 设疑解答导入, 直接 导入, 悬念导入等。导入方式都必须和要学习的知识 密切相关, 并能激起学生的好奇心和求知欲。

下面以《塑料成型工艺与模具设计》课程中的“注 射成型工艺条件”一节为例介绍教学目标的导入。首 先向学生展示下面几幅图片或者给学生这几件实物 产品, 请学生观察塑件产品成在的问题, 分析产生的 原因。并提出如下问题:

(1) 观察下列塑件存在哪些质量问题?

（2）在模具设计、制造、设备都正常的情况 下, 是哪些因素造成了产品的质量问题?

（3）如何高效的获得合格的塑件产品? 


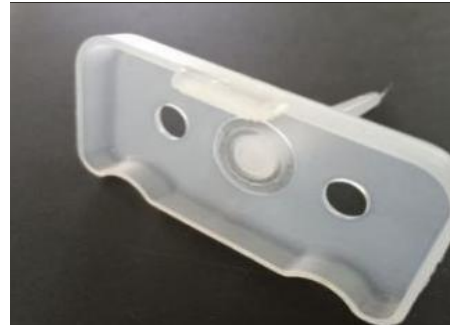

图 2 合格塑件

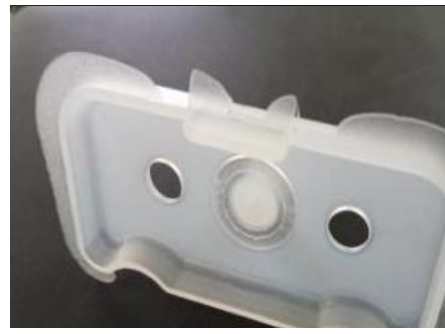

图 3 带飞边的塑件

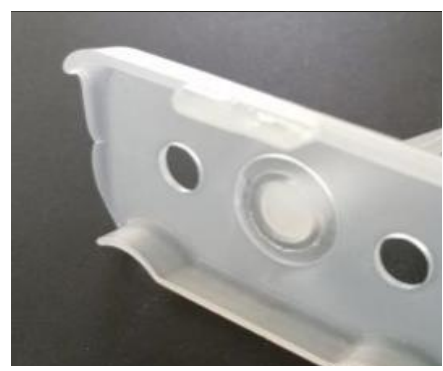

图 4 缺料的塑件

要回答以上问题需达成以下教学目标:

知识目标: 掌握注射成型的工艺参数; 掌握各参 数对塑料制品成型质量的影响?

能力目标: 观察能力, 分析能力, 动手能力 (通 过实验验证工艺参数对塑件质量的影响), 合理制定 注射工艺参数的能力。

情感目标: 沟通能力, 协作能力, 归纳总结能力。

\section{2. 教学目标的展开}

教学目标导入后, 对目标的实现展开教学活动, 这是目标教学法最关键、最重要的环节。采取何种教 学方法和手段来实现教学目标, 应根据具体的教学内 容和学生的情况合理、科学的选择。教学目标展开常 用以下几种教学方式, 教学过程中可灵活运用:

（1）精讲点拨。“精讲” 就是根据教学目标抓住 知识点, 讲解重点, 剖析难点, 释疑共性问题 [5], 对 学生容易掌握的基本知识点可以一带而过, 对每节中 的重点、难点、疑点等学生难于理解的重要的知识, 教师必须对此 “精讲”。要用通俗易懂的, 容易理解的 语言向学生进行阐述。让学生在尽量短的时间内掌握 知识的要点。“点拨” 就是洞悉学生思维, 发现学生疑 难, 及时指点和引导, 利用启发、诱导学生积极思考,
起到 “拨云见日”、“点石成金”、“茅塞顿开” 的作用。 使学生对所学知识理解的深, 理解的透, 掌握的牢。

(2) 独立探索。围绕教学目标让学生自主学习, 通过教材、参考资料、网络等资源收集教学目标所要 求的资料, 对知识进行理解、消化、归纳、整理。然 后教师再进行归纳总结。主要是培养学生利用各种资 源, 收集、整理资料, 获取知识并独立思考的能力。

(3) 情景设置。创设与学生所学的内容相接近的 现实情景环境, 把学生引入到现实问题的情景中, 要 求学生通过某知识点来解决。培养学生对所学知识的 运用能力和解决问题的能力, 为培养现场工程师打基 础。

(4) 提问解答。教师围绕教学目标提出一系列问 题, 让学生带着问题自学相关内容, 然后对每一个问 题进行抽问, 学生可以相互补充, 然后教师进行补充、 完善和总结, 对于比较难的问题可以启发、引导学生 完成作答。这种教学方法可以使学生从被动听变为主 动学, 活跃了课堂气氛, 调动了学生学习的积极性。 在提问时要注意问题难易相结合, 问题要有层次的递 进过程, 问题要有连贯性以及问题所涉及的知识面要 广, 尽量避免提深僻难懂的问题, 使学生无所是从, 从而失去学习的兴趣。

(5) 实验、实践训练。在课程设置中就要将实验、 实践内容纳入正规的教学计划中, 围绕教学目标, 展 开实验、实践教学, 让学生动手操作, 在操作过程中 解决教学目标提出的问题。这种教学方法需要有相应 的实验设备, 可以培养学生的动手能力、观察能力、 分析能力。应用型本科特别重视实践教学环节, 是培 养现场工程师的有力途径。

(6) 讨论交流。教师根据教学目标提出问题, 学 生通过自学得到自己的答案, 然后进行小组交流、讨 论, 对答案进行补充、完善, 最后形成统一答案, 再 进行小组间交流，共同探讨 ${ }^{[5]}$ 。教师根据每组的答案 进行分析、点评, 最后形成科学、合理的认识。这种 教学方法可以培养和锻炼学生的交流沟通能力、合作 能力、组织能力等, 增强团队意思。

（7）现代先进的教学资源。充分利用现代网络资 源, “超星”、“雨课堂”、“云课堂”、“网络精品课程”、

“视频”、“线上线下课堂” 等, 来充实和丰富教学内 容。开拓学生视野, 丰富学生的知识面。

对上面提到的 “注射成型工艺条件”一节的教学 过程, 可以采用以下的教学方法来实现教学目标。首 先通过 “精讲点拨” 学习该部分的理论知识, 接着进 行 “分组讨论”, 确定成型该塑件的工艺参数 (注射压 力、注射温度、注射时间)。然后分组在注射成型实验 设备上按照讨论确定的参数进行 “实验”, 观察成型产 品的质量是否满足设计要求, 根据产品的成型情况调 整工艺参数, 达到产品完全合格为止。最后对实验结 果进行 “归纳总结”, 得出工艺参数对塑件产品质量影 响的结论。通过实验我们会发现不同的参数都可以得 
到质量合格的产品, 三个参数是相互协调、相互制约 的关系, 如果设置的参数不合理就得不到合格的塑件 产品。我们需要质量合格且用时最短的那组参数来进 行生产以提高效率。

\section{3. 教学过程的把控}

在教学目标实施过程中必须坚持以教师为主导, 学生为主体, 围绕教学目标这条主线展开教学, 引导 学生积极思考, 认真学习, 注重学生能力的培养。让 学生观察, 合作, 辩论, 反思。通过观察, 发现问题; 通过合作, 取长补短; 通过辩论, 明晰思路; 通过反 思, 拨乱反正 ${ }^{[6]}$ 。但在教学过程中可能出现偏离主题, 冷场或激烈争论等各种各样的问题。如果教学中由于 知识的延伸引入了新的知识点, 且学生非常有兴趣, 但偏离了教学目标这一主线, 要及时采取合理有效的 方式、方法引导学生回归到教学目标这条主线上来, 不要沉迷于新的知识点而忘了主题。对于冷场要根据 造成冷场的原因采用相应的措施使气氛活跃起来, 如 果是提出的问题太难, 可以采用引导法、提示法、类 比法、讨论法等引导学生思考。如果是争论激烈且逐 渐偏离主题, 就要及时的终止讨论, 并给出合理的结 论。总之要把控好教学的节奏和进度, 否则就会造成 课堂松散, 无组织、无纪律, 达不到教学目标, 完不 成教学任务。

\section{6. 教学目标的检验与评价}

教学目标的本质是作为教学行为的规范和准则, 其制定是否合理, 需要通过教学来进行检验与评价, 然后对教学目标进行修改、完善, 逐步提高。教学目 标的检验与评价包括课堂上的检验和课后的评价。在 教学过程中教师要密切关注学生的思维状态, 注重信 息反馈, 掌握学生对教学目标的达成度, 即检验教学 效果。根据学生的状态及时分析、总结, 调整语言, 调整教学方法, 调动学生学习的积极性。在课堂上对 教学效果的检验可以通过提问、课堂小练习、小测验 和学生交谈等方式了解教学效果, 对出现的问题及时 进行补救。课后可以利用超星、慕课等线上资源对没 有搞清楚的问题进一步学习, 也可以与老师进行线上 交流，完成线上相应的作业、测验、考试等。使学生 对知识进一步加深和巩固, 提高教学目标的达成度。 教学的最终目的是让学生学到知识, 增长才能。

课后要对本讲课进行完整的教学评价, 对三个教 学目标的达成度进行打分, 并对达成目标所采用的教 学方法和手段是否适合, 是否最优进行分析。客观地 评价我们在教学过程中取得的成绩和存在的问题, 从 而对教学过程进行相应的调整。对学生易于接受, 能 提高学生学习积极性的我们要继续发扬, 对不足的要 立即改进。只有这样才能踏踏实实地提高教学质量, 提高学生的综合素质和竞争能力。

教学目标是多重的, 分层次的, 目标的达成并不 与教学完全同步。有的目标可以通过几节课的学习就
能直接达成, 如知识目标可以通过课堂教学、自学来 达成, 相对简单、容易, 也便于检验, 评价。能力目 标、情感目标的达成则可能需要一学期、一学年有的 甚至整个大学时期, 他是通过对教学活动、实践活动 等的凝练, 通过日积月累间接形成的更高层次的素质， 也更难于检验和评价的, 但我们不能就此不进行检验 和评价。应该针对具体的情况制定相应的检验和评价 机制。对于知识目标, 可以通过提问、测验、考试等 方式来检验教学目标的达成度; 对于能力目标、情感 目标可以通过考试、辩论、实验、创新等来检验和评 价。

\section{7. 结论}

目标教学法是一种有效的教学方法, 它突破了传 统的教学模式, 通过解决学生的一些实际问题来实现 学生对知识的掌握, 大大提高了学生学习的积极性和 主动性。通过目标教学法学习的学生, 他们的动手能 力、解决实际问题的能力都有很大的提高。在整个教 学过程中既发挥了教师的主导作用又体现了学生的 主体作用, 充分地展示了现代教育“以能力为本”的价 值取向, 使课堂教学的质量和效益得到更大幅度的提 高。对以培养 “现场工程师” 为目标的应用型本科院 校不适为一种最有效的教学方法。可以说, 目标教学 法是师生共同完成目标, 共同取得进步的教学方法。

\section{REFERENCES}

[1] Higher Education Law of the People's Republic of China (2018 amendment)

[2] goal-based approach https://baike.baidu.com/item

[3] Xu Bingwen. Yeung Yuk Biu. Objective teaching method: A probe into classroom teaching mode. Vocational Education, 2006(08) : 115-117.

[4] I use several leading-in methods in teaching https://wenku.baidu.com/

[5] Dai Chunshan [ J ] . A new attempt at goal-based approach. Chinese and foreign entrepreneurs, 2015(29) : 210 .

[6] Cheung Ying, on the basic practice of the guiding link in the "Target Teaching Method", Journal of learning 2017 No. 27 